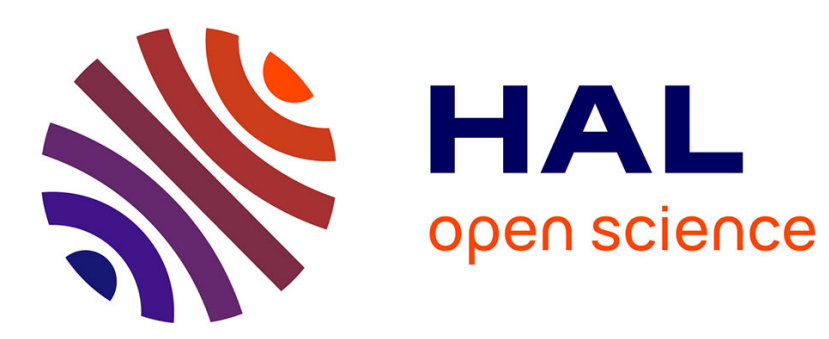

\title{
Catalyse électrochimique à l'aide de catalyseurs immobilisés sur support organique ou minéral
}

J. Devynck, F. Bedioui

\section{To cite this version:}

J. Devynck, F. Bedioui. Catalyse électrochimique à l'aide de catalyseurs immobilisés sur support organique ou minéral. Journal de Physique IV Proceedings, 1994, 04 (C1), pp.C1-131-C1-146. 10.1051/jp4:1994109 . jpa-00252451

\section{HAL Id: jpa-00252451 https://hal.science/jpa-00252451}

Submitted on 1 Jan 1994

HAL is a multi-disciplinary open access archive for the deposit and dissemination of scientific research documents, whether they are published or not. The documents may come from teaching and research institutions in France or abroad, or from public or private research centers.
L'archive ouverte pluridisciplinaire HAL, est destinée au dépôt et à la diffusion de documents scientifiques de niveau recherche, publiés ou non, émanant des établissements d'enseignement et de recherche français ou étrangers, des laboratoires publics ou privés. 


\title{
Catalyse électrochimique à l'aide de catalyseurs immobilisés sur support organique ou minéral
}

\section{J. DEVYNCK et F. BEDIOUI}

Laboratoire d'Electrochimie Analytique et Appliquée, URA 216 du CNRS, Ecole Nationale Supérieure de Chimie de Paris, 11 rue Pierre et Marie Curie, 75231 Paris cedex 05, France

\begin{abstract}
Modification of electrodes by film deposit containing transition metal complexes is reviewed in the scope of application to selective electrocatalytic reactions. The selected organized microstructures are obtained from organic conducting polymers and mineral clays and zeolites. The characterization of the electrochemical properties of the entrapped complexes is described. Applications in electrocatalytic reductions, hydrogenations, electrocatalytic oxidations and oxidations by molecular oxygen catalyzed by manganese porphyrins are described and discussed.
\end{abstract}

\section{Introduction}

La recherche d'une amélioration des propriétés catalytiques de complexes métalliques vis-à-vis des réactions électrochimiques par immobilisation du catalyseur au voisinage de l'électrode se développe au rythme de l'apparition des nouveaux matériaux, conducteur ioniques, conducteurs électroniques ou semi-conducteurs dont disposent les électrochimistes.

L'immobilisation du catalyseur peut-être obtenue par des moyens variés, dont les plus utilisés sont :

- la modification de la surface métallique ou semi-conductrice constituant elle même le catalyseur

- l'adsorption ou la chimisorption de couches, généralement monomoléculaires ou peu épaisses du catalyseur

-le dépôt de film d'épaisseur variable dans lequel est retenu le catalyseur, film qui crée une nouvelle phase entre la surface métallique ou semi-conductrice et la solution contenant les substrats et les produits de la réaction.

L'incorporation de catalyseurs dans des films "multicouches" d'épaisseur variable, pouvant aller de quelques couches moléculaires jusqu'à l'échelle millimétrique est, sans conteste, l'un des domaines les plus florissants de l'électrocatalyse. Les avantages de tels systèmes à la fois par rapport aux catalyseurs monocouches et par rapport aux catalyseurs 
en solution sont bien connus :

- le signal électrochimique est plus important que dans le cas d'une monocouche : le taux de recouvrement global, $\Gamma_{\text {total }}$ (exprimé en moles par $\mathrm{cm}^{2}$ ) étant plus élevé.

- la présence du catalyseur au voisinage de l'électrode assure un meilleur contrôle du transfert électronique.

- la stabilité du catalyseur peut être améliorée

- l'effet catalytique est augmenté par le confinement du catalyseur dans un volume limité (augmentation des interactions substrat-catalyseur)

D'une façon plus générale, l'existence d'une couche permet de créer, autour du catalyseur un environnement "sur mesure", en fonction des réactions recherchées, conduisant ainsi à une meilleure sélectivité vis-à-vis des processus recherchés.

Le confinement du catalyseur présente cependant quelques inconvénients du fait de l'introduction d'une nouvelle phase dans laquelle la diffusion des espèces (réactifs, électrolytes, produits de la réaction) est toujours plus lente qu'en solution.

Par ailleurs, les applications en électrosynthèse nécessitent une stabilité chimique des matériaux qu'il est d'autant plus difficile d'obtenir que les structures catalytiques sont plus élaborées.

Les développements récents des électrodes modifiées ont donné lieu à de nombreuses mises au point (1-5). Notre objectif sera limité ici à la description et aux applications de systèmes modificateurs d'électrodes incorporant des catalyseurs du type complexe métallique $\mathrm{ML}_{\mathrm{x}}$. ( $\mathrm{L}=$ amine, bipyridine, terpyridine, phénanthroline, base de Schiff, ou porphyrine). Avec de tels complexes, l'effet catalytique de ces complexes se résume parfois simplement à une "catalyse rédox" ( au sens habituel du terme), mais, le plus fréquemment, la catalyse est " chimique" et met en jeu des interactions catalyseur-substrat qui se produisent dans un environnement moléculaire qu'il convient de bien maîtriser et de bien décrire pour assurer aux processus recherchés le maximum d'efficacité.

L'attention sera donc plus particulièrement portée vers les moyens qu'il est possible de mettre en oeuvre pour améliorer l'environnement du catalyseur en créant des "microstructures" évoquées plus haut.

On privilégiera également les systèmes qui ont donné lieu à des applications, soit à l'échelle préparative en électrosynthèse.

Deux grandes familles de modificateurs d'électrodes support de catalyseurs sont ainsi envisagés :

- les films polymères organiques et, en particulier les polymères conducteurs qui, du fait de leur mise en oeuvre relativement aisée, ont connus un développement considérable ces dernières années

- les films contenant, comme structures hôte du catalyseur, des composés inorganiques à microstructure organisée, du type lamellaire ou à cavités zéolithique. On verra en particulier, comment l'introduction au voisinage de l'électrode, de tels composés permet la création de véritables "microréacteurs", aptes à apporter les éléments de sélectivité supplémentaire par comparaison aux structures type polymère organique. 


\section{I - Electrodes modifiées par des films polymères conducteurs contenant un complexe métallique}

L'incorporation de complexes catalyseurs dans des films polymères, conducteurs ou non, concerne une grande variété de matériaux. Plusieurs revues récentes (1-3) recensent ces matériaux et les caractéristiques du transport de charge dans les films correspondants. Le mécanisme et la cinétique des phénomènes catalytiques mis en oeuvre dans les films, dont les principaux éléments ont été rappelés récemment (6) ne sera pas décrit.

\section{Formation et caractérisation du catalyseur immobilisé}

Le mode de formation du film dépend essentiellement de la nature du matériau et les méthodes sont extrêmement diversifiées (1-3). Le tableau 1 rassemble les principaux systèmes évoqués dans cette présentation. Cependant, c'est l'électropolymérisation du complexe qui offre l'éventail le plus vaste des possibilités et qui a été le plus fréquemment mis en oeuvre. Le procédé passe par le greffage préalable d'un groupement pyrrole sur la molécule complexante, suivi de l'électropolymérisation par balayage cyclique du potentiel ou par électrolyse à potentiel imposé.

La modification préalable du complexant par greffage de pyrrole a été mise en oeuvre, en particulier, avec les complexes de la bipyridine $(1,7,8)$ de la terpyridine (9) et de porphyrines (10-15) ou de cyclam (16).

Tableau I - Quelques exemples de polymères conducteurs modifiés par greffage d'un composé rédox ou d'un complexant d'un ion métallique utilisés en catalyse préparative

\begin{tabular}{|c|l|}
\hline POLYMERE CONDUCTEUR & \multicolumn{1}{|c|}{ CATALYSEUR } \\
\hline Polypyrrole N-substitué(PP) & $\begin{array}{l}\text { systèmes rédox: } \\
\text { viologène, quinone, ferrocène } \\
\text { complexes } M L_{n} \\
\text { L=bipyridine, phénanthroline, } \mathrm{NH}_{3} \\
\mathrm{~L}=\text { hétérocycle azoté porphyrine, cyclam }\end{array}$ \\
\hline polypyrrole 3 substitué (PP3) & $\begin{array}{l}\text { système rédox : quinone, ferrocène, } \\
\text { phénothiazine }\end{array}$ \\
\hline $\begin{array}{c}\text { polythiophène (PT) } \\
\text { polyméthylthiophène }\end{array}$ & système rédox : catenand \\
\hline
\end{tabular}

On notera que certains de ces complexes ont également été incorporés sous forme chargée, simplement par dopage $(17,18)$ ou encore, récemment, par échange d'ions sur des polypyrrole-ammonium quaternaire $(19,20)$.D'autres complexes sont directement électropolymérisables et ne nécessitent pas la fixation préalable sur un composé comme le pyrrole. C'est le cas, par exemple, de certaines porphyrines (21) ou de complexes de bases de Schiff (22-24).

Sur le tableau 1 sont également mentionnés les principaux systèmes rédox envisagés pour l'étude fondamentale des propriétés des films ou comme médiateurs rédox dans de nombreuses applications : il s'agit des systèmes quinone/hydroquinone, ferrocène/ferricinium viologène, ou phénothiazine, pour ne citer que les plus 
couramment employés. Ces systèmes consituent du fait de leur rapidité, de bons catalyseurs rédox.

Un exemple de formation par électropolymérisation d'une porphyrine-pyrrole de cobalt à une électrode de carbone vitreux dans l'acétonitrile est présenté sur la figure 1. Le voltammogramme cyclique montre les systèmes' caractéristiques $\mathrm{Co}$ (III)/Co(II) et $\mathrm{Co}$ (II) $/ \mathrm{Co}$ (I) respectivement à $+0,42 \mathrm{~V}$ et $-0,89 \mathrm{~V} / \mathrm{ECS}$ et deux couples de pics à 1,1 et $1,3 \mathrm{~V}$ correspondant au macro-cycle de la porphyrine. La formation du film polymère se traduit par l'augmentation de la hauteur des pics caractéristiques avec le nombre de balayage du potentiel. La polymérisation du pyrrole est observée à $\mathrm{Ep}_{\mathrm{a}}=+0,7 \mathrm{~V}$. La présence du film polymère contenant la porphyrine est attestée par le tracé des voltammogrammes d'une électrode modifiée dans une solution ne contenant pas le monomère. Les systèmes rédox caractéristiques de l'ion métallique sont, en général, peu affectés par la fixation sur l'électrode, preuve que l'environnement immédiat de la molécule n'est pas modifié et que les propriétés catalytiques sont, a priori, conservées. Ces conclusions sont applicables a la grande majorité des complexes incorporés dans des polymères conducteurs.

Il convient de noter que les couples rédox mis en jeu dans les réactions catalytiques apparaissent fréquemment dans une zone de potentiel où le polymère n'est pas conducteur (voir le schéma figure 2, applicable, par exemple, au cas des porphyrines de cobalt). Le polymère se comporte alors comme un "polymère rédox", non conducteur, dont l'exemple type le mieux étudié est formé à partir de films de Nafion dans lesquels sont incorporés des complexes par échange d'ions (25).

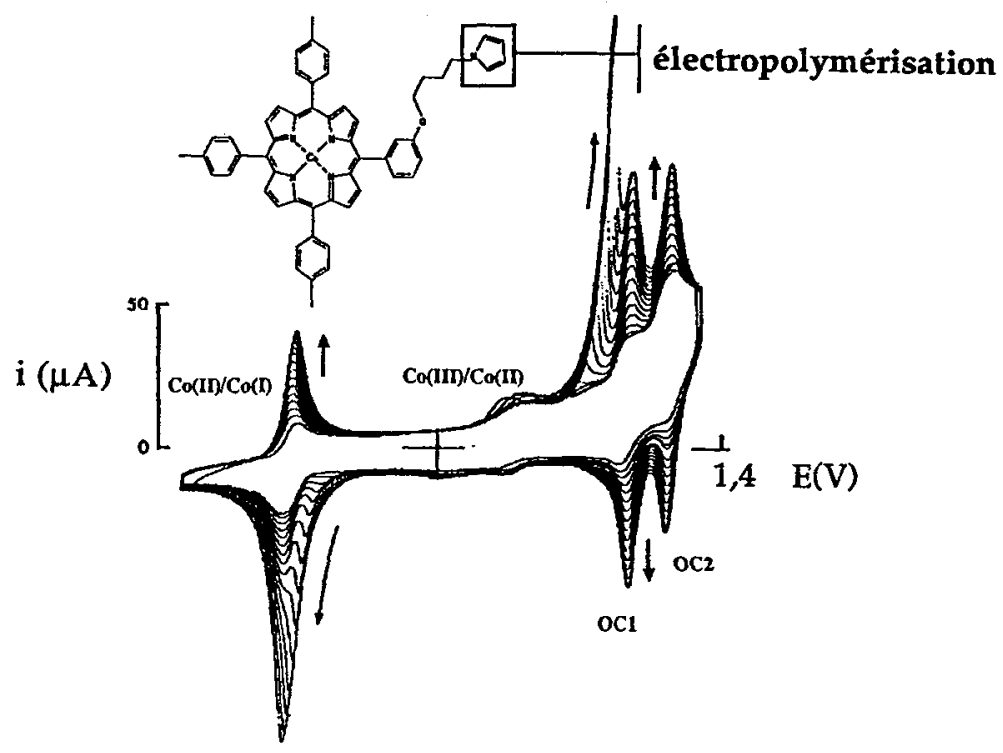

Figure 1 - formation, par électropolymérisation, d'un film de porphyrine de cobaltpolypyrrole. Electrode de carbone vitreux, milieu : acétonitrile + tétrafluoroborate de tétrabutylammonium $0,1 \mathrm{M}$; balayage cyclique du potentiel $(v=0,15 \mathrm{~V} / \mathrm{s})$. 


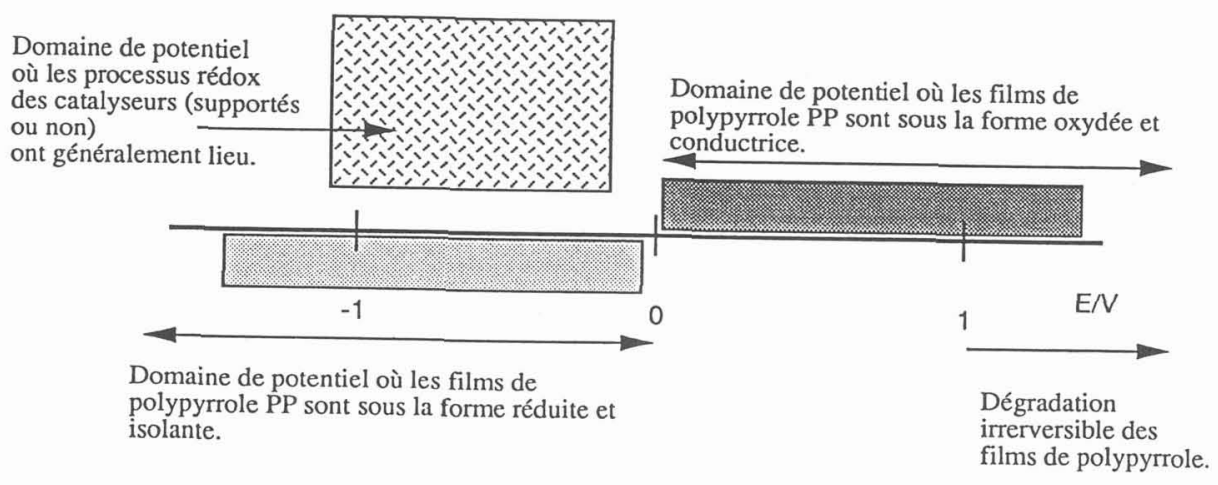

Figure 2 - propriétés électrochimiques d'un film de polypyrrole (PP) dans lequel est incorporé un composé rédox

On notera également qu'une faible fraction seulement du complexe présent dans le film polymère est électroactive et peut donc être impliquée dans les processus catalytiques, fraction variable, mais souvent reproductible, qui est rarement précisée par les auteurs.

L'origine de cette perte d'efficacité électrocatalytique du film polymère est très discutée. Dans le cas des électrodes polypyrrole-porphyrine, par exemple l'une des raisons est la diminution de la conductivité du film lorsque l'épaisseur augmente. Il a ainsi été montré qu'il existe une épaisseur optimale, en général assez faible, correspondant a une centaines de couches de porphyrines $(12,13)$, mais d'autres systemes catalytiques fonctionnent, à l'échelle préparative, en couche beaucoup plus épaisse (1).

\section{2- Amélioration des propriétés des systèmes catalytiques en vue des applications à l'électrosynthèse.}

\section{Création de microstructures à effet catalytique contrôlé}

L'amélioration des performances catalytiques des électrodes modifiées par des polymères conducteurs passe donc par la création d'un environnement du catalyseur plus élaboré, constituant une véritable "microstructure", dans laquelle on ajoute au polymère conducteur les éléments nécessaires. Parmi les modifications proposées sont les suivantes :

- copolymérisation du catalyseur avec une entité co-catalytique spécifique des réactions catalytiques envisagées (12). Il est connu qu'en catalyse d'oxydation des hydrocarbures par l'oxygène moléculaire et les métalloporphyrines, par exemple, l'utilisation d'un complexant dit "axial" du type pyridine est nécessaire. Ainsi, il est possible de mettre en évidence (par suite des variations des potentiels d'équilibre des couples rédox $\mathrm{Co}$ (III)/Co(II) et $\mathrm{Co}$ (II)/Co(I)) une complexation axiale "interchaine" du complexe à l'état Co(III)porphyrine par la pyridine dans le copolymère poly(CoTPP + pyridine).

- Incorporation d'agrégats métalliques dans le polymère. Cette opération conduit à ajouter aux effet catalytiques des complexes ceux des métaux divisés. Elle a conduit, dans le cas des polythiophènes, par ex., à une modification notable de la conduction (26). Dans le cas des polypyrroles, elle fournit de bons catalyseurs d'hydrogénation (27). 
- Création d'interactions spécifiques entre le polymère contenant le catalyseur et des entités en solution (28). L'activation du catalyseur greffé peut passer par l'ajustement du pouvoir complexant de la solution. L'utilisation des complexes du nickel à l'état réduit $\mathrm{Ni}(\mathrm{O})$, par exemple, nécessite l'amélioration de leur stabilité du fäit de leur forte réactivité. Cette meilleure stabilité peut être atteinte par la création d'un environnement "souple" autour du centre métallique pouvant conduire à une complexation supplémentaire du cation supporté dans le polymère par de la bipyridine en solution. A titre d'exemple, il a été montré que les performances électrochimiques et la stabilité des polymères préparés à partir du complexe $\mathrm{NiL}_{3}\left(\mathrm{BF}_{4}\right)_{2}$ ( $\mathrm{L}=$ bipyridine-pyrrole) sont effectivement améliorées soit par un ajout de bipyridine en solution (environ 2.10-5 M), soit par l'électropolymérisation du complexant $\mathrm{L}$ seul suivie par l'incorporation du cation $\mathrm{Ni}$ (II) par ajout de $\mathrm{NiCl}_{2}$ en solution (environ $10^{-4} \mathrm{M}$ ).

- Dépôt d'un film de perméabilité sélective à la surface du polymère conducteur.

On peut rendre sélectif l'accès aux sites catalytiques en protégeant le film polymère contenant le catalyseur par un second film, de perméabilité sélective. C'est ainsi qu'un film de polypyrrole contenant une porphyrine de nickel utilisé comme élément sensible dans le dosage électrochimique de $\mathrm{NO}$ est protégé des interférences de $\mathrm{NO}_{2}^{-}$par un film de Nafion, imperméable aux anions (29).

- Fixation de complexes chiraux

La fixation de complexes catalyseurs présentant un centre d'assymétrie permet d'espérer l'introduction d'une stéréosélectivité dans les réactions d'électrosynthèse. Quelques systemes mettant en oeuvre le polypyrrole ou les polythiophènes (3) sont décrits, mais aucun n'a été développé en électrosynthèse.

\section{3 - Applications à la catalyse électroassistée préparative des films polypyrrole}

Bien que l'électrocatalyse de réactions organiques ait été une des principales motivations dans le développement des électrodes modifiées par des polymères conducteurs fonctionnalisés, il n'existe que relativement peu d'exemples de systèmes mis en jeu à l'échelle préparative. Les propriétés catalytiques des films sont le plus souvent caractérisées par les modifications des voltammogrammes qui en résultent, en particulier dans le cas de la réduction de l'oxygène ou des ions $\mathrm{H}^{+}$.

En ce qui concerne les réactions organiques, c'est le polypyrrole qui constitue le support de choix. Des exemples sont décrits dans la revue de DERONZIER et MOUTET (1). Le tableau II regroupe l'essentiel des applications.

\section{hydrogénations}

Les polymères conducteurs, particulièrement le polypyrrole et les dérivés du polythiophènes, sont d'excellents supports de catalyseurs à base de métaux très divisés. La combinaison d'un métal dispersé ( $\mathrm{Pt}, \mathrm{Pd}$ ) et d'un couple rédox est très efficace pour les hydrogénations (27), par exemple. Une étude systématique de l'influence des principaux paramètres ( nature et concentration du métal, structure du polymère) a permis de mettre en évidence les points suivants :

- Dans le cas de substrats comportant plusieurs fonctions réductibles, la régiosélectivité dépend de la nature du métal.

- Le rendement électrique augmente avec la quantité de métal dans le cas des molécules difficilement hydrogénables. 
- Le rendement électrique de l'hydrogénation des molécules encombrées dépend de la réticulation du polymère.

\section{oxydations électocatalytiques}

Les oxydations menées à l'échelle préparative avec des structures complexes associant le polypyrrole à des systèmes rédox et à $\mathrm{Ru}_{2}$ constituent de bons catalyseurs d'oxydation des alcools (30) et présentent des turnover parfois très élevés.

\section{oxydation biomimétique par l'oxygène moléculaire}

Cette oxydation, catalysée par des porphyrines de fer ou de manganèse, constitue un exemple très démonstratif de l'intérêt des films polymères en catalyse.

Le principe de l'activation de l'oxygène moléculaire par les porphyrines de manganèse en solution organique, et la nature schématique des différents intermédiaires "oxygénés" intervenant dans les réactions de catalyse d'oxydation d'hydrocarbures d'une part et de phénols d'autre part sont représentés sur le schéma ci-dessous. Ce schéma, inspiré du mode de fonctionnement du cytochrome P450, montre la nécessité de régénérer les entités catalytiques par réduction; son application dans le cas d'une régénération électrochimique a été proposé, en phase homogène, par MURRAY(31) et il a été montré qu'il était transposable sur électrode modifiée $(32,33)$.

\section{Tableau II}

Quelques exemples de catalyse de réactions électrochimiques préparatives à l'aide d'électrodes modifiées

\section{Réductions}

\begin{tabular}{|ccc|}
\hline réaction & catalyseur & référence \\
\hline Réduction de $\mathrm{RX}$ et $\mathrm{R}^{\prime} \mathrm{X}_{2}$ & $\mathrm{PP}+$ viologène & 34 \\
\hline Hydrogénation & $\begin{array}{c}\mathrm{PP}-\mathrm{Viologène}+\mathrm{métal}(\mathrm{Pd}, \mathrm{Pt}) \\
\mathrm{PP}-\text { ammonium }+ \text { métal } \\
\mathrm{PP}-\mathrm{M}(\mathrm{Bipy})(\mathrm{M}=\mathrm{Rh}, \mathrm{Pd})\end{array}$ & $\begin{array}{c}27 \\
35\end{array}$ \\
\hline Réduction de $\mathrm{CO}_{2}$ & $\mathrm{PP}-\mathrm{Re}(\mathrm{Bipy})(\mathrm{CO})_{3}(\mathrm{Cl})$ & 36,37 \\
\hline réduction des nitrites & $\mathrm{PP}-\mathrm{Fe}($ porphyrine $)$ & 38 \\
\hline
\end{tabular}

oxydations

\begin{tabular}{|ccc|}
\hline Oxydation d'alcools & $\begin{array}{c}\text { PP-Ru (Bipy) }+\mathrm{RuO}_{2} \\
\text { PP-Ru(bipy)(terpy) }\end{array}$ & 30 \\
\hline $\begin{array}{c}\text { Oxydation par } \mathrm{O}_{2} \\
\text { Phénols, alcanes, alcènes }\end{array}$ & PP-M (Porphyrine) & 32,33 \\
\hline
\end{tabular}

abréviations et symboles : PP : polypyrrole ; PP-M(complexe) : polypyrrole fonctionnalisé par un complexe du métal $\mathrm{M}$; bipy : bipyridine ; terpy : terpyridine ; RX : dérivé halogéné. 
Ainsi, les tests catalytiques d'oxydation d'hydrocarbures et de phénols substitués ont été réalisés avec des électrodes de feutre et de tissus de carbone et de graphite de "grande" surface ( 2 a $4 \mathrm{~cm}^{2}$ ) recouverts de poly (pyrrole-porphyrine de manganèse). La régénération des espèces catalytiques est réalisée par imposition du potentiel $\mathrm{E}=-0.5 \mathrm{~V} / \mathrm{ECS}$ à l'électrode modifiée. Les résultats obtenus pour les réactions modèles d'oxydation d'hydrocarbures tels que les cyclooctènes, cyclohexènes, cyclooctanes, indanes et tétraline par l'oxygène moléculaire électrocatalysées par les films polymères de porphyrine de manganèse ont prouvé de manière indiscutable la possibilité de transposition en phase supportée de ces systèmes biomimétiques modèles $(32,33)$. De l'ensemble des résultats, et par comparaison à ceux publiés dans la littérature en phase homogène, il ressort principalement que l'efficacité du catalyseur (en terme de "turnover") est très largement améliorée lorsque celui-ci est fixé sur l'électrode. De plus, ces résultats montrent que la transformation des produits est réalisée avec de bons rendements faradique et chimique. Les électrodes sont stables au cours du temps.

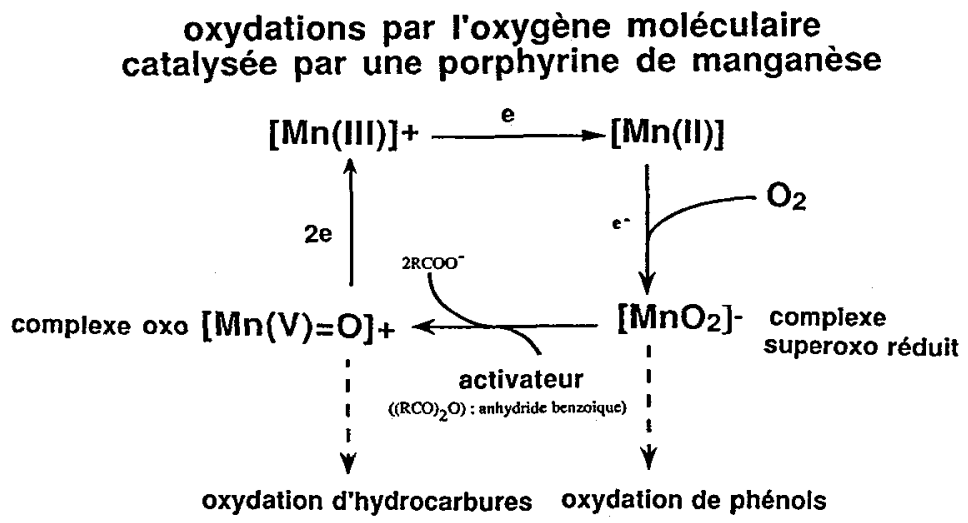

\section{II - Electrodes modifiées par des films contenant des complexes fixés sur supports minéraux}

\section{1 - Incorporation de complexes dans les structures minérales}

La fixation de complexes catalyseurs sur des polymères organiques décrite plus haut permet d'obtenir une sélectivité réactionnelle liée à l'environnement du catalyseur dans le film; elle n'offre cependant pas (ou peu) de sélectivité de taille et de forme du fait, essentiellement, du caractère peu stucturé du film organique.

Le problème de la sélectivité de forme et de taille peut être abordé en incorporant le catalyseur dans un environnement de structure mieux définie. Plusieurs approches sont possibles :

- La construction d'une structure organique autour du centre catalytique, étudiée par exemple, par de nombreux auteurs dans le cas des porphyrines modèles de cytochrome.

- L'incorporation du complexe dans une structure de forme et de taille bien déterminée. Les structures minérales lamellaires, tubulaires ou à cavité bien définie offrent cette possibilité.

Les types de structure envisageables sont schématisés sur la figure 3.

La structure minérale peut définir une " cage " de taille différente selon la nature des élements constitutifs. L'exemple-type est donné par les zéolithes dans lesquels l'arrangement des "cages" de sodalite définit une cavité intérieure ( dite "supercage") dans 
laquelle il est possible de placer un catalyseur pour effectuer une réaction. Le site catalytique n'est accessible qu'aux substrats de taille adaptée. Parmi les zéolithes courantes, la faujazite est celle qui présente les supercages les plus grandes ( diamètre intérieur de $13 \AA$ ).

La structure peut être tubulaire : les tubes définissent des canaux, généralement reliés entre eux, dans lesquels se place le catalyseur. Un bon exemple est donné par la zéolithe ZSM-5. La distance entre les canaux est de l'ordre de $10 \AA$.

Dans les composés à structure lamellaire, le catalyseur vient se placer entre les feuillets. La distance inter-feuillet est généralement fonction de nombreux paramètres : hydratation, nature et taille des réactifs intercalés, etc.. L'exemple type est la montmorillonite, dont la distance interfeuillet est de $4 \AA$ à l'état sec et de $18 \AA$ à l'état hydraté.

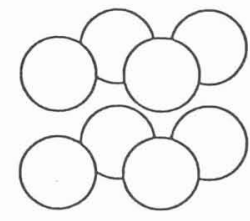

cages

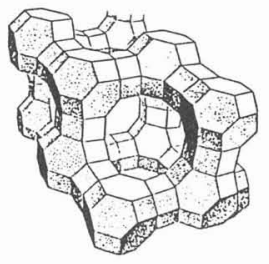

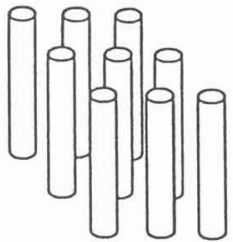

canaux

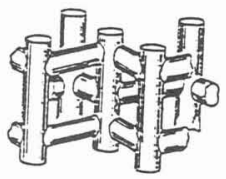

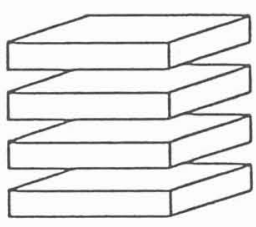

feuillets

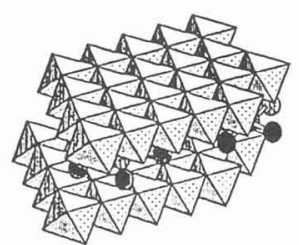

Figure 3 - Matériaux de confinement moléculaire : topologie des différents types de structure-hôte et exemples. cage : zéolithe type faujazite : canaux : zéolithe type ZSM-5 ; feuillets : dihydroxyde lamellaire $\left[\mathrm{Mg}_{6} \mathrm{Al}_{2}(\mathrm{OH})_{16}\right]^{2+}$.

Dans tous les cas, la taille du site d'accueil permet d'insérer des ions métalliques ou des molécules de petite taille; les complexes envisagés ici ne peuvent être logés que dans les zéolithes du type Faujazite ou dans les stuctures lamellaires de taille variable. Le degré de précision de l'environnement du catalyseur est évidemment fonction de la rigidité de la structure.

Les composés de ces deux familles possédent des propriétés d'échangeurs d'ions qui facilitent l'incorporation des catalyseurs ou de leurs éléments constitutifs sous forme ionique.

\section{2 - Réalisation d'électrodes composites à base de sructures minérales.}

Les composés décrits ci-dessus ne sont pas conducteurs électroniques : leur utilisation comme modificateur d'électrode nécessite donc l'emploi d'un "liant" apte à assurer le transfert électronique. Le contact peut être assuré de différentes façons :

- Par l'incorporation des minéraux dans un film de polymère non conducteur comme le polystyrène, ou conducteur, comme le polypyrrole ou le polyacétylène (40) contenant éventuellement un médiateur rédox (41).

- Par la réalisation d'un mélange avec du graphite, ou avec une "pâte" de carbone 
Le transfert de charge est réalisé, soit par l'intermédiaire du "liant", soit par la mise en oeuvre de médiateurs rédox, ou par les deux processus à la fois. On trouvera de nombreux exemples dans la récente mise au point de BARD et MALLOUK (5).

\section{3 - Electrodes composites à base d'argile lamellaire}

La montmorillonite et les complexes lamellaires du même type comme la laponite sont parmi les stuctures minérales les plus utilisées pour constituer des électrodes composites depuis les premiers travaux de BARD en 1982 (5).

En ce qui concerne plus précisément les porphyrines, la caractérisation électrochimique d'une porphyrine tétracationique du type TMPyP (figure 4) dans une montmorillonite montre que les systèmes rédox sont peu affectés par l'intercalation (42). La même remarque peut être faite au sujet d'une porphyrine anionique ( du type tétrasulfonate) intercalée dans une structure lamellaire comme le dihydroxyde d'aluminium et de magnésium (43).

Exemple d'application : catalyse électroassistée par une porphyrine intercalée dans une structure lamellaire du type montmorillonite.

La sélectivité introduite par la montmorillonite dans des réactions électroassistées peut être appréciée lors de l'oxydation d'hydrocarbures par l'oxygène moléculaire décrite ci-dessus. La comparaison des rendements d'oxydation du cyclooctane ou de l'adamantane obtenus avec la porphyrine en solution ou fixée sur un support type montmorillonite montre une oxydation préférentielle du cyclooctane lorsque le catalyseur est fixé sur support (Tableau III). Dans ces expériences, le système catalytique : Montmorillonite - Porphyrine est maintenu en suspension dans une solution d'acétonitrile contenant l'électrolyte-support, la base axiale et le cocatalyseur.

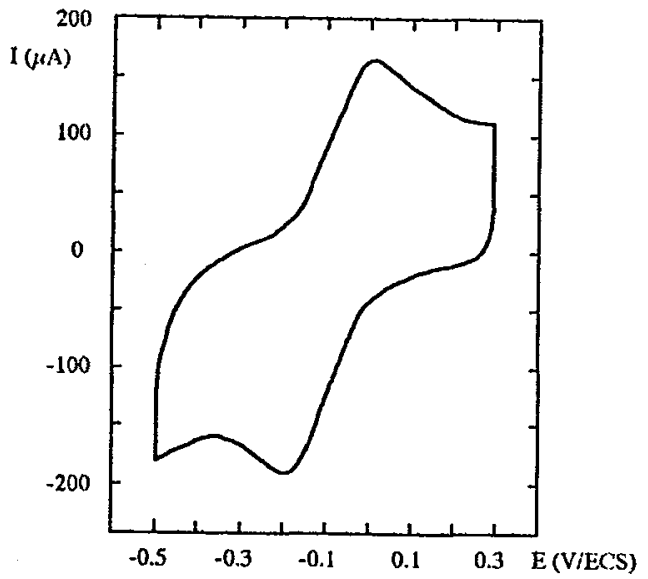

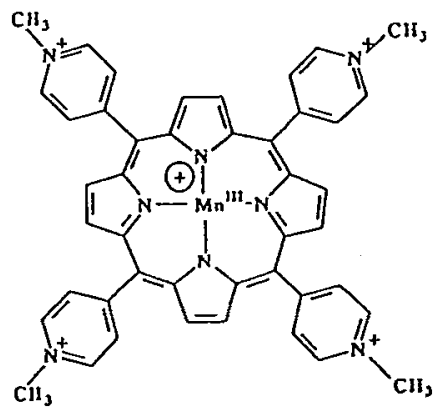

$[\mathrm{Mn}(\mathrm{III}) \mathrm{TMPyP}]^{4+}$

Figure 4 - Voltammétrie cyclique de la MnTMPyP4+ intercalée dans la montmorillonite. Electrode composite graphite-montmorillonite ; milieu : DMSO + $\mathrm{TBABF}_{4} 0,1 M(42)$. 
La régénération du catalyseur est obtenue en maintenant le potentiel de l'électrode à $-0,3 \mathrm{~V}(44)$. Le résultat est en accord avec les observations faites lorsque le catalyseur est régénéré chimiquement (45).

La sélectivité n'est cependant pas très grande du fait de l'existence de sites catalytiques hors de la structure lamellaire dont l'importance est étroitement tributaire du mode de préparation du catalyseur.

Tableau III - Oxydation d'un mélange de cyclohexane et d'adamantane par l'oxygène catalysée par une porphyrine de manganèse fixée sur montmorillonite (en suspension dans le milieu réactionnel) ou en solution.

\begin{tabular}{|c|c|c|c|c|}
\hline CATALYSE & CATALYSEUR & $\begin{array}{c}\text { PRODUITS } \\
\text { D'OXYOATION } \\
\text { DU } \\
\text { CYCLOHEXANE } \\
\text { (mmol, total) }\end{array}$ & $\begin{array}{c}\text { SOMME DES } \\
\text { PRODUITS } \\
\text { D'OXYDATION } \\
\text { DE } \\
\text { LADAMANTANE } \\
\text { (mmol, total) }\end{array}$ & $\begin{array}{c}\text { RAPPORT } \\
\text { produits } \\
\text { Cyclohexane } \\
\text { produits } \\
\text { Adamantane }\end{array}$ \\
\hline Electrochimique & $\begin{array}{c}\text { Montmorillonite } \\
\text { en suspension }\end{array}$ & 7,1 & 46,7 & 0,15 \\
\cline { 2 - 5 } & $\begin{array}{c}\text { Porphyrine } \\
\text { en Solution }\end{array}$ & 0,5 & 22,5 & 0,02 \\
\hline \multirow{2}{*}{+ Phimique } & $\begin{array}{c}\text { Montmorillonite } \\
\text { en suspension }\end{array}$ & 3,2 & 8,4 & 0,38 \\
\cline { 2 - 5 } & $\begin{array}{c}\text { Porphyrine } \\
\text { en Solution }\end{array}$ & 1,2 & 7 & 0,17 \\
\hline
\end{tabular}

\section{4 - Catalyseurs fixés sur zéolithe}

La rigidité de la structure des zéolithes implique un comportement du matériau différent de celui des argiles lamellaires dans la mesure où, d'une part, l'accès des sites de taille bien déterminée que sont les "supercages" n'est possible qu'aux espèces de taille adaptée aux ouvertures, et, d'autre part, où les molécules ou ions de grande taille peuvent se trouver emprisonnés dans la structure zéolithique.

Les petites molécules ou ions peuvent ainsi former des complexes qui, du fait de leur taille, sont encapsulés à l'intérieur des supercages. On dispose alors de véritables "microréacteurs", constitués par l'ensemble : cavité zéolithique + complexe "encapsulé" qui présentent une très grande sélectivité de taille et de forme.

Les premiers composés encapsulés sont des complexes Cobalt-base de Schiff décrits par HERRON et coll. en 1985 et utilisés pour l'oxydation des phénols par l'oxygène moléculaire (46). Depuis cette date, de nombreux autres systèmes ont été décrits, en particulier par OZIN (47) et mis en oeuvre dans l'oxydation chimique des alcanes. Les modes de préparation de complexes "encapsulés" ont été variés : il est possible d'introduire le complexe par simple échange d'ions, de préparer le complexe "in situ" en introduisant l'ion métallique et les ions ou molécules complexantes ( exemple : complexes de la bipyridine, de la phénanthroline ou de base de Schiff), de préparer le complexant luimême à l'intérieur des cavités (exemple : phthalocyanines) ou encore de synthétiser la zéolithe autour du complexe $(48,49)$. Un exemple de complexe encapsulé est donné sur la figure 5 . 
En ce qui concerne plus particulièrement les porphyrines, la taille de la molécule ne permet pas l'accès aux supercages par les ouvertures. Une synthèse "in situ" a été proposée mais la preuve de la présence de la porphyrine à l'intérieur des cavités n'a pas été apportée (50).

Outre son intérêt lié à la sélectivité de taille, l'encapsulation présente un avantage essentiel dans la catalyse d'oxydation par l'oxygène, en empêchant la désactivation du catalyseur par formation d 'oxo-dimères.
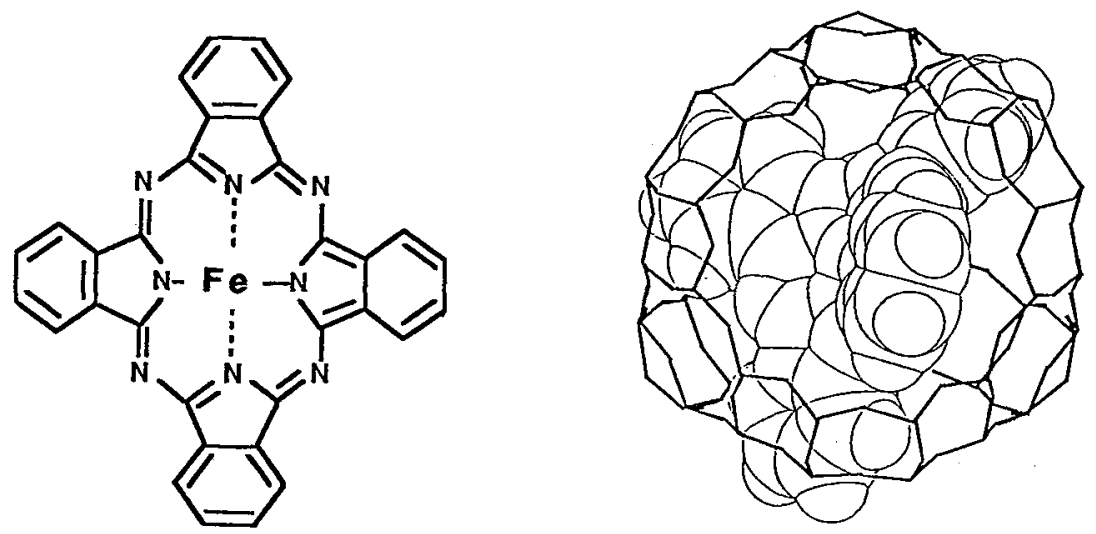

Figure 5 - Représentation d'une phthalocyanine de fer encapsulée dans la supercage d'une faujazite

\section{5 - Electrodes composites à base de zéolithes}

Les électrodes composites à base de zéolithe sont obtenues de la même façon que les électrodes à base d'argile lamellaire, et les constituants des couples rédox sont généralement fixés par échange d'ions. La capacité d'échange d'une faujazite, par exemple, est d'environ $4 \mathrm{meq} / \mathrm{g}$, du même ordre de grandeur que les résines échangeuses d'ions à base de polystyrène.

De nombreux ions et complexes électroactifs ont été fixés sur des zéolithes pour constituer des électrodes modifiées. Les principaux systèmes sont décrits dans la mise au point de ROLISON (4). Cependant, la caractérisation des complexes encapsulés par des méthodes électrochimiques est récente $(48,51,52)$. Un exemple de voltammétrie d'un complexe de base de Schiff dans une faujazite est représenté sur la figure 6 ; il met en évidence les couples rédox de l'ion métallique à un potentiel sensiblement identique à celui où ils sont observés en solution et fait apparaître des modifications qui pourraient être spécifiques d'interactions entre le complexe et la structure zéolithique (51).

\section{Application des électrodes à base de zéolithe en catalyse électroassistée}

Malgré l'intérêt des systèmes à base de zéolithe, il n'existe encore que peu d'exemples de systèmes catalytiques électroassistés mettant en oeuvre ces matériaux.

Une premiere application concerne l'oxydation des phénols par l'oxygène moléculaire, catalysée par une porphyrine de manganèse fixée sur zéolithe et régénérée électrochimiquement (53). La porphyrine est fixée par échange d'ions et il a été montré qu'elle n'occupait que des sites extérieurs à la cavité zéolithique ( $0,5 \%$ des sites cationiques). Dans cet exemple, la sélectivité n'est pas différente de celle observée avec le catalyseur en solution ou sur un support du type polymère. 


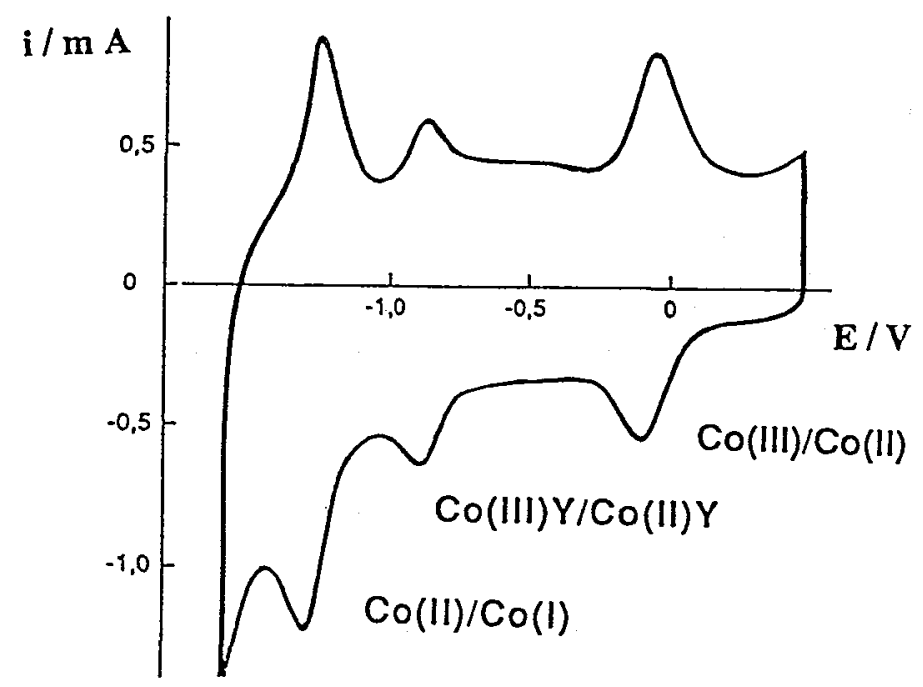

Figure 6 - Voltammogramme de Co-Salen encapsulé dans une faujazite (51)

En ce qui concerne les complexes encapsulés, l'exemple suivant montre : 1) que la catalyse électroassistée est possible et 2 ) que la sélectivité de taille peut être mise en évidence.

La catalyse de la réduction électrochimique des halogénures d'aryle par les complexes Ni-bipyridine est bien connue et décrite par ailleurs (54). Cette réaction passe par la formation d'un complexe de $\mathrm{Ni}(0)$ dont on peut mettre en évidence la formation et la disparition par voltammétrie cyclique. La figure 7 montre le voltammogramme d'une électrode composite graphite / $\mathrm{Ni}\left(\right.$ Bipy) $_{3}{ }^{2+}$ encapsulé dans une faujazite. Le couple $\mathrm{Ni}(\mathrm{II}) / \mathrm{Ni}(0)$ apparaît à $-0,7 \mathrm{~V}$ en l'absence de $\mathrm{RX}$. En présence de $\mathrm{RX}$, la différence entre les courbes (b) et $(c)$ montre que le bromure de triphénylméthyle n'a pas accès au site catalytique.

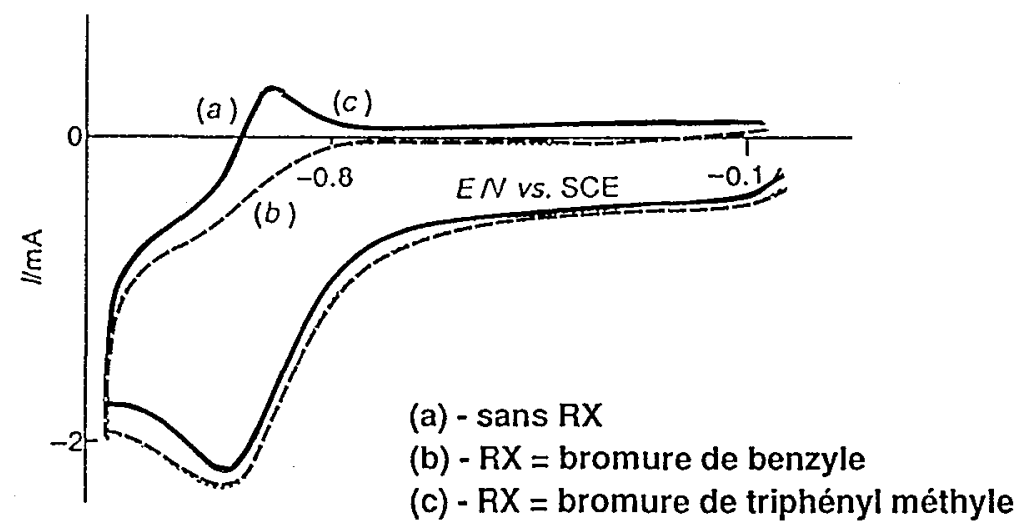

Figure 7 - Voltammogramme d'une électrode de graphite/Ni(bipy) $3 /$ zéolithe dans l'acétonitrile + Perchlorate de tétraéthylammonium $0,1 \mathrm{M}$. (a) : sans $R X ;(b)$ : en présence de $R X=$ bromure de benzyle ; (c) : en présence de $R X=$ bromure de triphénylméthyle. (54) 


\section{Conclusion}

La fixation de catalyseurs de réactions d'électrosynthèse dans un film " épaiș" à la surface d'une électrode présente, dans la plupart des exemples donnés, de réels avantages par comparaison avec la catalyse électroassistée en phase homogène. L'électropolymérisation de complexes polymérisables ou, d'une façon plus générale, la mise en oeuvre de polymères conducteurs s'avère une voie très efficace pour former des films de composition parfaitement contrôlée permettant la constitution de microstructures adaptées aux réactions recherchées. Il apparaît cependant évident que le caractère conducteur du polymère est rarement mis à profit dans la réaction catalytique elle-même du fait de l'intervention des réactions catalytiques spécifiques du complexe incorporé hors du domaine de conduction du matériau.

Les films de polypyrrole en particulier se sont montrés suffisamment stables, dans les domaines des potentiels explorés, pour la réalisation de réactions à l'échelle préparative. Les exemples de catalyse d'hydrogénation et d'oxydation électrocatalytique ou d'oxydation par l'oxygène sont, de ce point de vue, particulièrement concluants.

Des progrès dans la sélectivité des réactions peuvent encore être accomplis en améliorant l'environnement moléculaire du catalyseur et l'utilisation de structures minérales de géométrie parfaitement contrôlée constitue une voie de développement qui n'en est encore qu'à ses débuts. Les travaux en cours visant, en particulier, à analyser finement les modes de transfert électronique dans les systèmes à base de zéolithe trouvent leur application en électrocatalyse aussi bien qu'en photochimie. Par ailleurs, les travaux sur la catalyse à l'aide de complexes supportés pourraient bénéficier des recherches sur les solides macroporeux présentant des cavités de taille plus grande que celle de la faujazite et de stabilité suffisante. La comparaison des ouvertures de tels solides et de la taille de complexes ou des substrats mis en jeu, schématisée sur la figure, 8 illustre bien ces nouvelles potentialités.

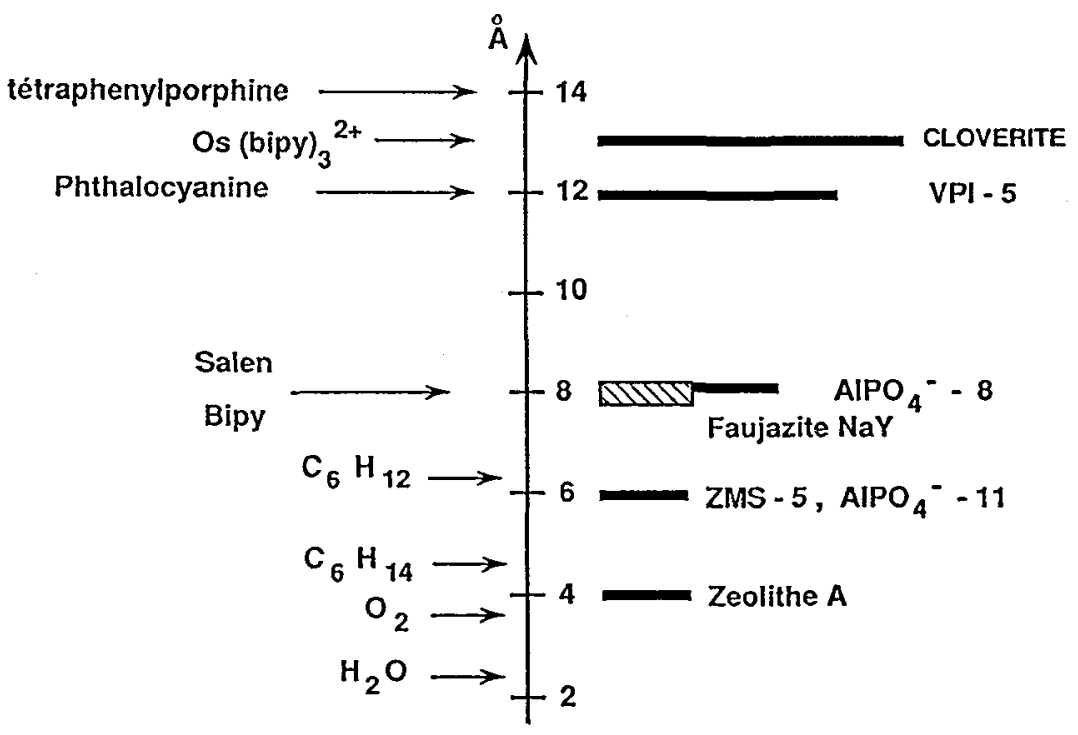

Figure 8 - comparaison de la taille des ouvertures des zéolithes ou de nouvelles structures macroporeuses et $d u$ diamètre apparent de quelques molécules-substrat, de ligands et de complexes. 


\section{Références}

1- DERONZTER, A. et MOUTET, J.C., Acc. Chem. Res., 22 (1989) 249.

2 - HEINZE, J., Topics Current Chem,152 (1990) 1.

3 - $\quad$ MERZ, A., Topics Current Chem., 152 (1990) 49.

4 - $\quad$ ROLISSON, D. R., Chem. Rev., 90 (1990) 867.

5 - BARD, A.J. et MÁLLOUK, T.J., dans " Molecular Design of Electrode Surface", (MURRAY, R.J. ed.), Wiley and Son, N.Y., (1992)

6 - ANDRIEUX, C.P. et SAVEANT, J.M., dans "Molecular Design of Electrode Surface", (MURRAY, R.J. ed.), Wiley and Son, N.Y., (1992)

7- DAIRE, F., BEDIOUI, F., DEVYNCK, J. et BIED-CHARRETON, C., J. Electroanal. Chem., 224 (1987) 95.

8 - $\quad$ EAVES, J.G., MUNRO, H.S., et PARKER, D.,Inorg. Chem., 26 (1987) 644

9 - BIDAN, G., DIVISIA-BLOHORN, B., KERN, J.M. et SAUVAGE, J.P., J. Chem. Soc., Chem. Comm., (1988) 723.

10 DERONZIER, A. et LATOUR, J.M., J. Electroanal. Chem., 224 (1987) 295.

11 BEDIOUI, F., MERINO, A., DEVYNCK, J. , MESTRES, C.E. et BIEDCHARRETON, C., J. Electroanal. Chem., 239 (1988), 433. ARMENGAUD, C., MOISY, P., BEDIOUI, F. DEVYNCK, J. et BIEDCHARRETON, C., J. Electroanal. Chem., 277 (1990) 197.

13 BEDIOUI, F., VOISIN, M., DEVYNCK, J. et BIED-CHARRETON, C., J. Electroanal. Chem., 297 (1991) 257.

14 DERONZIER,A., DEVAUX, R., LIMOSIN, D. et LATOUR, M., J. Electroanal. Chem.,319 (1991) 347.

15 RAMACHANDRAIAH, G., BEDIOUI, F., SERRAR, M. , DEVYNCK, J. et BIEDCHARRETON, C., J. Electroanal. Chem.,319 (1991) 395. COLLIN, J.P. et SAUVAGE, J.P., J. Chem. Soc., Chem. Comm.,(1987) 1075.

17 OKABAYASHI,K., IKEDA, O. YOSHIDA, N et TAMURA, H., J. Electroanal. Chem.,191 (1985) 157.

18 BEDIOUI, F., HINNEN, C., BONGARS, C., BIED-CHARRETON, C., J. Electroanal.Chem., 207 (1986) 87.

19 DE GREGORI, I., CARRIER, M., DERONZIER, A., MOUTET, J.C., BEDIOUI, F. et DEVYNCK, J., J. Chem. Soc., Faraday Trans., 88 (1992) 1567.

20 BEDIOUI, F, BOUYER, Y., SOREL, C., DEVYNCK, J., COCHE-GUERENTE, L., DERONZIER, A. et MOUTET, J.C., Electrochim. Acta, sous presse.

21 YOUNATHAN, J.N., WOOD, K.S. et MEYER, T.J., Inorg. Chem., 31 (1992) 3280 et références citées.

22 HORWITZ, C.P. et MURRAY, R.W., Mol. Cryst. Liqu. Cryst., 160 (1988) 389.

23 AUDEBERT, P., CAPDEVIELLE, P. et MAUMY, M., New J. Chem.,15 (1991) 235. BEDIOUI, F., GUTIERREZ-GRANADOS, S. et DEVYNCK, J., J. Electroanal. Chem.,301 (1991) 267. TOURILLON, G. ef GARNIER, F., J. Phys. Chem., 88 (1984), 5281 et réf. citées. COCHE, L. et MOUTET, J.C., J. Am. Chem. Soc., 109 (1988) 6887 et COCHE, L., EHUI, B., LIMOSIN, J. et MOUTET, J.C., J. Org. Chem., 55 (1990) 5905. 
LABBE, E., BEDIOUI, F. et DEVYNCK, J., J. Electroanal. Chem., 274 (1989) 271. MALINSKI, T. et TAHA, Z., Nature, 358 (1992) 676.

COSNIER, S., DERONZIER, A. et MOUTET J.C., Inorg. Chem., 27 (1988) 2389. CREAGER, S.E., RAYBUCK, S. et MURRAY, R.W., J. Am. Chem. Soc., 108 (1986) 3225.

MOISY,P., BEDIOUI, F. , ROBIN, Y et DEVYNCK, J., J. Electroanal. Chem.,250 (1988) 191.

BEDIOUI, F., GUTIERREZ-GRANADOS, S., DEVYNCK, J. et BIEDCHARRETON, C., New J. Chem., 15 (1991) 939.

COCHE, L., DERONZIER, A. et MOUTET, J.C., J. Electroanal. Chem., 198 (1986) 187.

DE OLIVEIRA, I.M.F., MOUTET, J.C., et HAMAR-THIBAULT,S., J. Electroanal. Chem., sous presse.

DE OLIVEIRA, I.M.F., MOUTET, J.C. et VLACHOPOULOS, N., J. Electroanal. Chem., 291 (1990) 243.

DERONZIER, A., MOUTET, J.C. et SAINT-AMAN,E., J. Electroanal. Chem., sous presse.

O'TOOLE, T.R., SULLIVAN, B.P., BRUCE, M.R.M., MARGERUM, D. MURRAY, R.W. et MEYER, T.J.,J. Electroanal. Chem., 259 (1989) 217.

DE GIOVANI, W.F. et DERONZIER, A., J. Electroanal. Chem., 337 (1992) 285. BEIN, T. et EUZEL, P., Angew. Chem., Int. Ed. Engl., 28 (1989) 1692.

LI, Z. et MALLOUK, T.E., J. Phys. Chem., 91 (1987) 643.

\section{GAILLON, L., BEDIOUI, F., DEVYNCK, J. BATTIONI, P., BARLOY, L. et} MANSUY, D., J. Electroanal. Chem., 303 (1991) 283.

GAILLON, L., BEDIOUI, F., DEVYNCK, J. et BATTIONI, P., J. Electroanal. Chem., 347 (1993) 435.

4 GAILLON, L., BEDIOUI, F., BATTIONI, P. et DEVYNCK, J., J. Mol. Catal., 78 (1993) 123.

5 BARLOY, L., BATTIONI, P. et MANSUY, D., J. Chem. Soc., Chem. Comm., (1990) 1365.

6 HERRON, N., TOLMAN, C.A. et STUCKY, G.D., Inorg. Chim. Acta, 100 (1985) 135.

OZIN, G.A. et GIL, C., Chem. Rev., 89 (1989) 1749.

BEDIOUI, F., DE BOYSSON, E., DEVYNCK, J. et BALKUS Jr, K.J., J. Electroanal. Chem., 315 (1991) 313 et références citées.

9 BALKUS Jr, K.J., DOUGLAS-HARGIS ef C. KOWALAK, S. dans :

"Supramolecular Architecture", (T. BEIN, ed.), ACS Symp. Ser., 499 (1992) 347. NAKAMURA, M, TATSUMI, T. et TOMINAGA, H., Bull. Soc. Chim. Jap., 63 (1990) 3334.

1 BEDIOUI, F., DE BOYSSON, E., DEVYNCK, J. et BALKUS Jr, K.J., J. Chem. Soc., Farad. Trans., 87 (1991) 3831.

2 GAILLON, L., SAJOT, N., BEDIOUI, F., DEVYNCK, J. et BALKUS Jr., K.J., J. Electroanal. Chem., 345 (1993) 157.

3 DE VISMES, B., BEDIOUI, F., DEVYNCK, J., PERREE-FAUVET, M. et BIEDCHARRETON, C., Nouv. J. Chim., 10 (1986) 81.

4 MESFAR, K., CARRE, B., BEDIOUI, F, et DEVYNCK, J., J. Mat. Chem, sous presse. 